\title{
Serum insulin-like growth factor-1 as a potential marker for MDD diagnosis, its clinical characteristics, and treatment efficacy validation: data from an open-label vortioxetine study
}

\author{
Oleg A. Levada ${ }^{1}$, Alexandra S. Troyan ${ }^{1 *}$ (i) and Irina Y. Pinchuk ${ }^{2}$
}

\begin{abstract}
Background: IGF-1 is an essential neurotrophin produced peripherally and in the brain. Impairments in the brain IGF-1 concentrations might be responsible for some aspects of major depressive disorder (MDD) pathogenesis, whereas peripheral IGF-1 could have the marker value. We aimed: 1) to compare serum IGF-1 levels in MDD patients and healthy controls $(\mathrm{HC}) ; 2$ ) to elucidate possible associations between changes in IGF-1 expression and crucial characteristics of the current depressive episode, MDD course; 3) to evaluate IGF-1 dynamics after 8 weeks' vortioxetine treatment.
\end{abstract}

Methods: Seventy-eight MDD patients (according to DSM-5) and 47 HC were enrolled. Serum IGF-1, psychopathological (MADRS, CGI) and neuropsychological parameters (PDQ-5, RAVLT, TMT-B, DSST) were analyzed in all subjects at admission and 48 patients after 8 weeks' vortioxetine treatment. AUC-ROCs were calculated to determine if the value of serum IGF-1 could separate MDD patients from HC. Multiple regression models were performed to explore relationships between IGF-1 and depressive episode's symptoms.

Results: MDD patients had significantly higher serum IGF-1 levels than HC (228 (183-312) ng/ml vs 153 (129-186) ng/ml, $p<0.0001)$. IGF-1 had a good diagnostic value for predicting MDD in the whole sample with AUC of $0.820(p<0.0001)$. For a cutoff of $178.00 \mathrm{ng} / \mathrm{ml}$, the sensitivity and specificity were 83 and 71\%, respectively, and the number needed to misdiagnose was 5 , indicating that only 1 of 5 tests give an invalid result. Among MADRS items, only reported sadness, inner tension, and concentration difficulties were significantly positively associated with serum IGF-1 concentrations. Vortioxetine treatment significantly attenuated IGF-1 levels and improved all psychopathological, neuropsychological parameters.

\footnotetext{
* Correspondence: troian@zmapo.edu.ua

'State Institution "Zaporizhzhia Medical Academy of Postgraduate Education Ministry of Health of Ukraine", 20 Winter Boulevard, Zaporizhzhia 69096, Ukraine

Full list of author information is available at the end of the article
}

(c) The Author(s). 2020 Open Access This article is licensed under a Creative Commons Attribution 4.0 International License, which permits use, sharing, adaptation, distribution and reproduction in any medium or format, as long as you give appropriate credit to the original author(s) and the source, provide a link to the Creative Commons licence, and indicate if changes were made. The images or other third party material in this article are included in the article's Creative Commons licence, unless indicated otherwise in a credit line to the material. If material is not included in the article's Creative Commons licence and your intended use is not permitted by statutory regulation or exceeds the permitted use, you will need to obtain permission directly from the copyright holder. To view a copy of this licence, visit http://creativecommons.org/licenses/by/4.0/. The Creative Commons Public Domain Dedication waiver (http://creativecommons.org/publicdomain/zero/1.0/) applies to the data made available in this article, unless otherwise stated in a credit line to the data. 
(Continued from previous page)

Conclusions: Significant associations between IGF-1 levels and hypothymia, anxiety, and cognitive disturbances may indicate a pathogenic role of IGF-1 for the mentioned symptoms. We assume that the activity of the cerebral-hepatic axis increases in response to insufficient IGF-1 brain expression in MDD patients, whereas, vortioxetine treatment restores cerebral IGF-1 concentrations and, consequently, decreases its compensatory production by the liver.

Trial registration: registered at ClinicalTrials.gov (NCT03187093). First posted on 14th June 2017.

Keywords: IGF-1, Depression, Cognitions, Vortioxetine

\section{Background}

Depression remains one of the crucial areas of scientific and applied psychiatric research over the past decades [1]. Such a situation is explained by the leading place of major depressive disorder (MDD) in the structure of psychiatric morbidity, as well as its social and economic consequences [2, 3]. MDD diagnosis is still based on clinical criteria [4], while modern treatment strategies are aimed at the deciphered biological pathways of the disorder [5]. Among them, special attention is paid to neuroplasticity [6-9]. Abnormal neural plasticity has been related to changes in the expression of neurotrophic factors [10]. Alterations in the signal pathways of neurotrophins can be considered as possible targets of therapy. At the same time, changes in their levels in body fluids can be used as biological markers of MDD diagnosis and treatment effectiveness.

Insulin-like growth factor-1 (IGF-1) is an essential neurotrophin that is produced both peripherally [11] and in the brain $[12,13]$. It is involved in cell growth, differentiation, and maturation (through DNA synthesis and mitosis stimulation), in metabolic (i.e., glucose uptake and protein production) [13] and neuroplastic processes (synapses formation, neurotransmitters releasing, and exciting of neurons) [12]. Due to IGF-1 pleiotropic functions, it has been assumed that impairments in the IGF-1 system might be responsible for some aspects of MDD pathogenesis [13], as well as peripheral IGF-1 levels could have the marker value [14]. According to our recent review [14], the majority of studies demonstrate higher concentrations of peripheral IGF-1 in MDD patients compared to healthy controls (HC). Moreover, sufficient body of evidence suggests that antidepressant treatment decreases IGF-1 levels [14]. Nevertheless, it is still unclear whether the increased peripheral expression of the neurotrophin can be considered as a marker of the actual depressive episode (DE) itself, its structural domains (affective, cognitive or somatic disturbances), severity, clinical subtype or mentioned elevation is associated with the characteristics of the course of MDD (duration, number of episodes, etc.). Solving those questions could shed light on some aspects of MDD pathogenesis and contribute to improving the diagnosis of the disorder.
Taking into account the above arguments, our goals were: 1) to compare peripheral levels of IGF-1 in MDD patients and $\mathrm{HC} ; 2$ ) to elucidate possible associations between changes in IGF-1 expression and crucial characteristics of the current DE and MDD course; 3) to evaluate the dynamics of the neurotrophin levels after 8 weeks of treatment with vortioxetine.

\section{Methods \\ Participants and procedures}

This was a case-control study, which included 125 participants aged 18 to 65 years. Seventy-eight outpatients diagnosed with MDD according to DSM-5 criteria were recruited through Zaporizhzhia Regional Clinical Psychiatric Hospital, Ukraine. Eligibility criteria for the study inclusion were detailed elsewhere [15]. At study entry, all the patients received no actual antidepressant medication. Subjects were excluded if they had any other psychiatric diagnosis, high suicidal risk, substance dependence/abuse over the past year, significant neurological disorders, head trauma, unstable medical conditions, history of endocrine diseases, psychotic features in the current episode, high risk for a hypomanic switch. HC $(n=47)$ with no current psychiatric disorder were age-, sex-, and educationmatched to MDD subjects. $\mathrm{HC}$ were excluded based on the use of medications and/or illicit drugs; the intake of alcohol within $48 \mathrm{~h}$ of the study visit; and the presence of an unstable medical condition, which could affect cognitive function.

After confirmation of eligibility, participants provided written informed consent to take part in the study and attended a baseline visit to complete all evaluations (overall 125 subjects). The assessments were repeated in 48 patients after 8 weeks of treatment with flexiblydosed vortioxetine 10-20 mg per day (two participants were lost for follow-up and not included). Only those patients receiving vortioxetine treatment were included in the analyses after treatment. The study flowchart is depicted in Fig. 1.

The study was approved by the local ethics committee and performed in accordance with the ethical standards laid down in the 1964 Declaration of Helsinki and its 


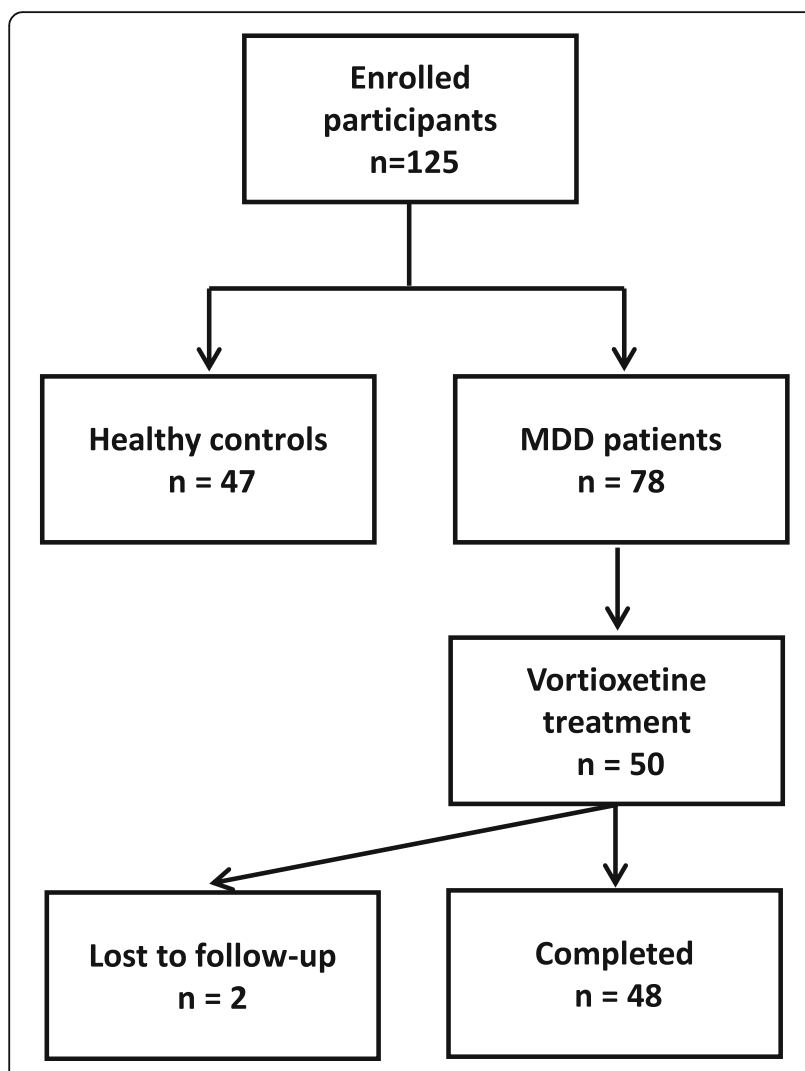

Fig. 1 The flowchart of participant disposition

later amendments and registered at ClinicalTrials.gov (NCT03187093).

\section{Clinical assessments}

Depression severity at baseline and changes after treatment were evaluated using MADRS [16] and Clinical Global Impression Severity (CGI-S) and Improvement (CGI-I) scales [17].

Subjective cognitive functioning was assessed using the Perceived Deficit Questionnaire-5 (PDQ-5), a 5-item self-report scale, which measures perceived difficulties of cognitive functioning. Each item ranges from 0 to 4, with higher scores reflecting greater severity [18].

\section{Neuropsychological assessments}

To characterize the cognitive functioning, a selected battery that included neuropsychological tasks covering the most impaired cognitive domains in MDD was administered to all participants. The tests were managed using paper and pencil. The instruments included:

1) Rey Auditory Verbal Learning Test (RAVLT), to evaluate immediate verbal memory, retroactive and proactive interference effects, delayed recall and recognition [19];
2) Trail Making Test B (TMT-B), to assess processing speed, executive function, i.e., set shifting [19];

3) Digit Symbol Substitution Test (DSST), to evaluate processing speed, executive function, learning, memory, attention, and concentration [19]..

\section{IGF-1 measurements}

In MDD patients and HC, IGF-1 concentration was measured in the morning under fasting conditions. Blood was drawn by a venous puncture between eight and eleven a.m. within the first 2 days after clinical assessments in 125 participants at baseline and 48 patients after 2 months of vortioxetine (10-20 mg per day) treatment. Blood was centrifuged at $3000 \mathrm{~g}$ for $10 \mathrm{~min}$, and the serum was stored at $-20{ }^{\circ} \mathrm{C}$ until further processing. IGF-1 was measured using the chemiluminescence immunoassay Immulite 2500 (Siemens AG, Germany) and Human IGF-1 Quantikine ELISA Kit (R\&D Systems Inc., Minneapolis, USA). As IGF-1 standard, the WHO IRR $87 / 518$ was used, which offers a measurement range with the Immulite test from 20 to $1600 \mathrm{ng} / \mathrm{ml}$.

\section{Statistical analysis}

Data analyses were carried out with the statistical package SPSS for Windows, version 20.0 (SPSS Inc., USA). The results were given as median and interquartile range or means and SDs or percentages, depending on the data. The statistical significance of between-group comparisons was determined using non-parametric and parametric criteria when appropriate (Mann-Whitney test, Wilcoxon test, chi-squared test, T-test). To identify potential covariates for analyses for IGF-1 levels, preliminary correlational analyses were conducted between IGF-1 concentration and demographic and clinical variables using Spearman's $r_{s}$. After that, the areas under the receiver operating characteristic curves (AUC-ROC) were calculated to determine if the value of serum IGF-1 level could separate MDD participants from HC. A cutoff was derived from the ROC curve to yield empirical optimal sensitivity and specificity. The number needed to misdiagnose [20] was calculated for this cutoff. For ROC analyses, the whole sample was also divided into five groups according to age $(18-24,25-34,35-44,45-$ $54,55-65$ years), as preliminary correlational analyses showed a significant negative association between age and IGF-1 concentrations. Afterward, multiple linear regression models were performed to explore potential relationships between IGF-1 levels and different symptoms of a DE (according to MADRS items). All four models included MADRS items and age, gender, level of education, and depression severity as covariates. The second model also included the number of the current DE; the third - included the duration of the current DE; the fourth - included both the number and length of the 
Table 1 Demographic, psychopathological, neuropsychological characteristics and serum IGF-1 levels in HC and MDD patients

\begin{tabular}{|c|c|c|c|}
\hline & $\begin{array}{l}\mathrm{HC} \\
n=47\end{array}$ & $\begin{array}{l}\text { MDD } \\
n=78\end{array}$ & $p$ \\
\hline \multicolumn{4}{|l|}{ Demographic characteristics } \\
\hline Women, n (\%) & $27(57.4 \%)^{\mathrm{a}}$ & $48(61.5 \%)$ & 0.65 \\
\hline Age, years ${ }^{*}$ & $37.8(12.3)^{b}$ & $38.2(11.9)$ & 0.84 \\
\hline Education, years ${ }^{*}$ & $15.2(2.1)^{b}$ & $14.5(1.9)$ & 0.60 \\
\hline \multicolumn{4}{|l|}{ Clinical assessments } \\
\hline \multicolumn{4}{|l|}{ Psychopathological } \\
\hline MADRS total score & $2(0-4)^{c}$ & $29(22-33)$ & $<0.0001$ \\
\hline CGI-S score & $1(1-1)^{c}$ & $4(4-5)$ & $<0.0001$ \\
\hline \multicolumn{4}{|l|}{ Patient-reported cognitive symptoms } \\
\hline PDQ-5 total score & $1(0-2)^{c}$ & $7(5-11)$ & $<0.0001$ \\
\hline \multicolumn{4}{|l|}{ Neuropsychological testing } \\
\hline RAVLT immediate recall total score & $64(58-69)^{c}$ & $49(43-56)$ & $<0.0001$ \\
\hline RAVLT proactive interference score & $8(6-9)^{c}$ & $6(5-7)$ & $<0.0001$ \\
\hline RAVLT retroactive interference score & $15(13-15)^{c}$ & $11(9-12)$ & $<0.0001$ \\
\hline RAVLT delayed recall score & $15(14-15)^{c}$ & $11(9-12)$ & $<0.0001$ \\
\hline RAVLT delayed recognition score & $15(15-15)^{c}$ & $14(14-15)$ & $<0.0001$ \\
\hline TMT-B (s) & $57(42-65)^{c}$ & 77 (64-93) & $<0.0001$ \\
\hline DSST number of correct symbols & $62(54-68)^{c}$ & $50(43-59)$ & $<0.0001$ \\
\hline IGF-1 serum level (ng/ml) & $153(129-186)^{c}$ & $228(183-312)$ & $<0.0001$ \\
\hline
\end{tabular}

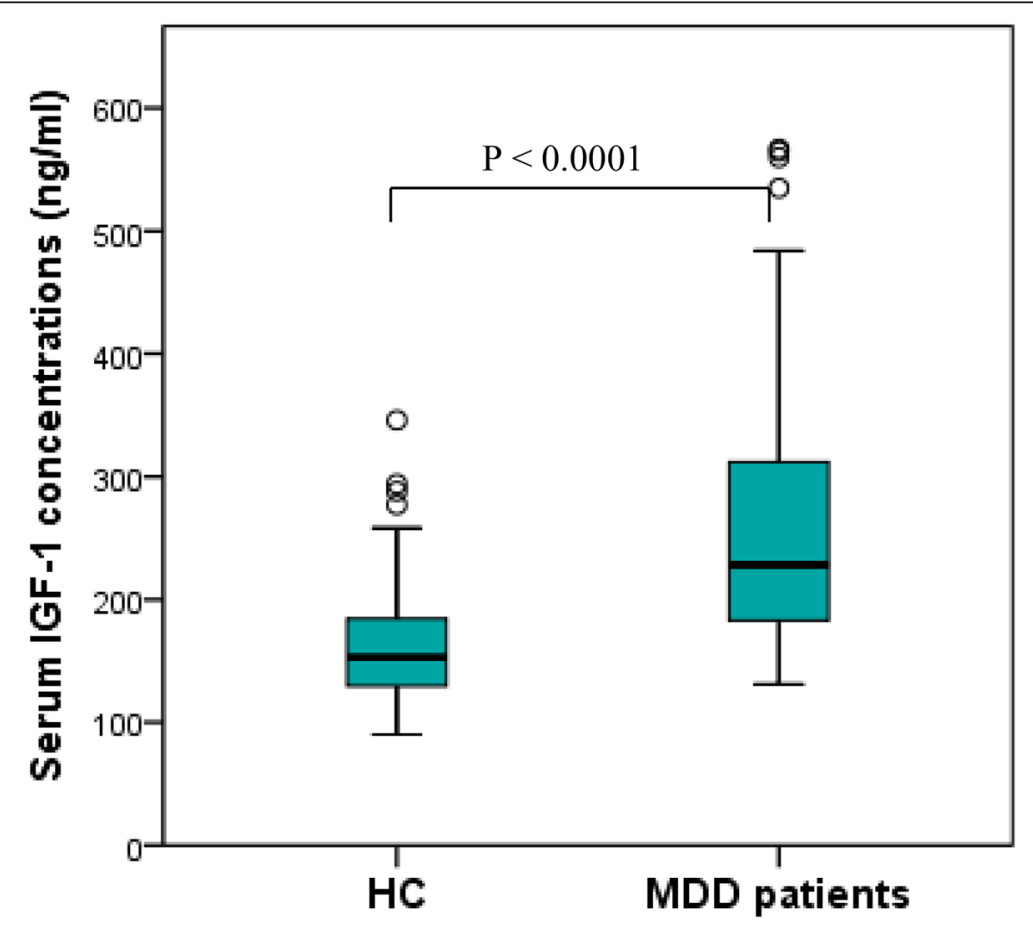

Fig. 2 Serum IGF-1 levels in HC and MDD patients 
current DE. Besides, multiple linear regression models were performed to explore potential relationships between IGF-1 levels and the performance of each cognitive test, adjusted for the same variables (age, gender, education, depression severity, number and duration of the current DE). Lastly, we compared IGF-1 levels of 48 patients at baseline and after 8 weeks of treatment using Wilcoxon test for dependent samples. Significance was set at $p<0.05$.

\section{Sample size determination}

To determine the minimum of participants per group to compare IGF-1 levels, we used the following formula: $2 \mathrm{SD}^{2}\left(\mathrm{Z}_{\alpha / 2}+\mathrm{Z}_{\beta}\right)^{2} / \mathrm{d}^{2}$, where $\mathrm{SD}=$ standard deviation, $Z_{\alpha / 2}=1.96$ at type 1 error of $5 \%, Z_{\beta}=0.842$ at $80 \%$ power, $\mathrm{d}=$ difference between mean values. A previous study revealed the following IGF-1 levels in MDD patients vs controls $157 \pm 40 \mathrm{mg} / \mathrm{ml}$ and $120 \pm 33 \mathrm{mg} / \mathrm{ml}$, respectively [21]. Therefore, a minimum of 18 people per group had to be included. For regression analyses, we used Green's rule, which requires a minimum of $104+\mathrm{k}$ subjects (where $\mathrm{k}$ is the number of predictors).

\section{Results}

Demographic and clinical characteristics of the sample

The main demographic and clinical features and IGF-1 levels of the comparison groups are depicted in Table 1. Surveyed cohorts were comparable in age, gender, and level of education. Besides the expected statistical difference in MDD patients and HC on MADRS and CGI-S scales, sufficient distinction in neuropsychological test performance was found between the comparison groups. MDD participants were significantly worse $(p<0.0001)$ in executive functioning (DSST, TMT-B scores), processing speed (DSST, TMT-B scores), set shifting (TMT-B), and all parameters of verbal memory (RAVLT subtests). Regarding serum IGF-1, we detected significantly elevated levels of the neurotrophin in MDD patients as compared to controls (Fig. 2).

Data are presented as median (upper-lower quartile) unless otherwise stated; "data are presented as means (SD); ${ }^{a}$ Chi-square test, controls vs MDD patients; ${ }^{\mathrm{b}} \mathrm{T}$ test, controls vs MDD patients; ${ }^{\mathrm{C}}$ Mann-Whitney U-test, controls vs MDD patients.

IGF-1 correlations with demographic, psychopathological and neuropsychological variables

Next, we performed a correlational analysis to determine possible associations between serum IGF-1 concentrations and demographic and clinical parameters in the whole sample (Table 2). We found a significant inverse correlation between serum IGF-1 levels and age and positive correlations between serum IGF-1 levels and the number, duration, and severity of the current DE.
Table 2 Spearman's correlation coefficients between demographic, psychopathological, neuropsychological characteristics and serum IGF-1 levels in MDD patients and HC

\begin{tabular}{|c|c|}
\hline Variables & $r_{s}$ \\
\hline Age, years & $-0.25 * *$ \\
\hline Number of DE & $0.31 * *$ \\
\hline Duration of DE, weeks & $0.42^{* *}$ \\
\hline Education, years & -0.07 \\
\hline CGI-S score & $0.51^{* *}$ \\
\hline PDQ-5 total score & $0.40^{* *}$ \\
\hline MADRS total score & $0.48^{* *}$ \\
\hline Apparent sadness & $0.45^{* *}$ \\
\hline Reported sadness & $0.50^{* *}$ \\
\hline Inner tension & $0.53^{* *}$ \\
\hline Reduced sleep & $0.30^{* *}$ \\
\hline Reduced appetite & $0.38^{* *}$ \\
\hline Concentration difficulties & $0.46^{* *}$ \\
\hline Lassitude & $0.36^{* *}$ \\
\hline Inability to feel & $0.47^{* *}$ \\
\hline Pessimistic thoughts & $0.53^{* *}$ \\
\hline Suicidal thoughts & $0.35^{* *}$ \\
\hline RAVLT immediate recall total score & $-0.34^{* *}$ \\
\hline RAVLT proactive interference score & $-0.23^{*}$ \\
\hline RAVLT retroactive interference score & $-0.37^{* *}$ \\
\hline RAVLT delayed recall score & $-0.32^{* *}$ \\
\hline RAVLT delayed recognition score & -0.05 \\
\hline TMT-B (s) & $0.40^{* *}$ \\
\hline DSST number of correct symbols & $-0.21^{*}$ \\
\hline
\end{tabular}

${ }^{*} p<0.05 ;{ }^{* *} p<0.01$

Additionally, we found that IGF-1 levels significantly correlated with the MADRS items and cognitive tests` scores.

\section{IGF-1 levels for MDD diagnosis}

The discriminating ability of serum IGF-1 elevation to separate MDD participants from $\mathrm{HC}$ was determined by ROC analysis. Figure 3 represents the ROC curves for serum IGF-1 to diagnose MDD. IGF-1 had a good diagnostic value for predicting MDD in the whole sample with AUC of $0.820(p<0.0001)$. For a cutoff of 178.00 $\mathrm{ng} / \mathrm{ml}$, the sensitivity and specificity were 83 and $71 \%$, respectively, and the number needed to misdiagnose was 5 , indicating that only 1 of 5 tests give an invalid result. For AUC analyses, the whole sample was also divided into five age groups, as preliminary correlational analyses showed a significant negative association between age and IGF-1 concentrations. Age ranges for the groups were as follows: Group 1-18-24 years old (HC: $n=7$, MDD: $n=8$ ); Group 2-25-34 years old (HC: $n=16$, 


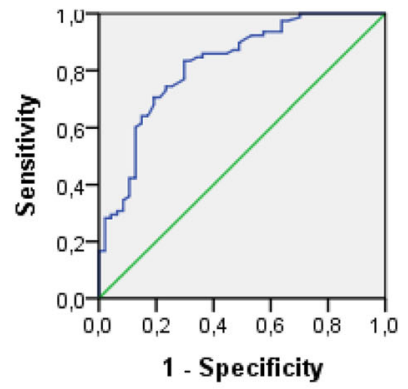

A

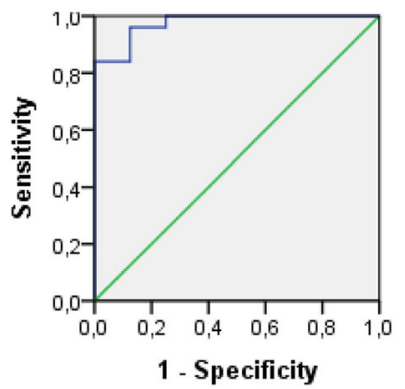

D

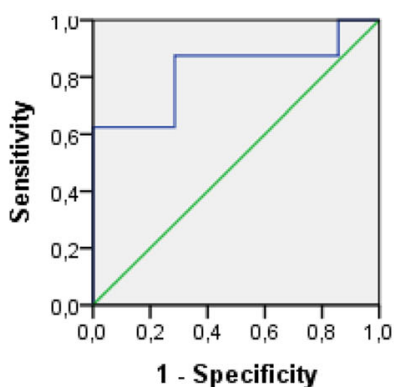

B

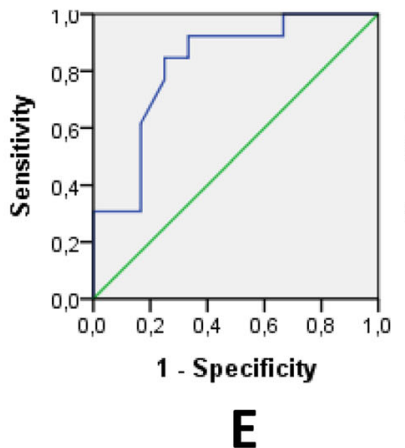

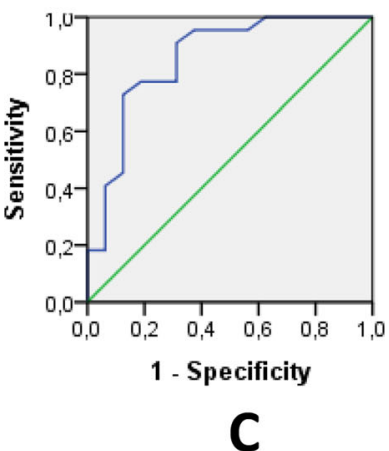

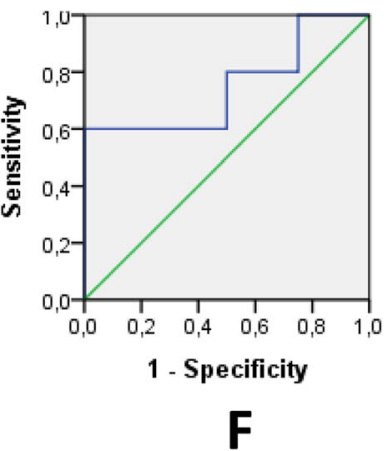

Fig. 3 ROC curves for serum IGF-1 level and MDD status. a ROC curve to diagnose MDD in all age groups (18-65 y.o.) ( $p<0.0001$, AUC: 0.820; sensitivity: $83 \%$ and specificity: $71 \%$ for a cutoff of $178.00 \mathrm{ng} / \mathrm{ml})$. b ROC curve to diagnose MDD in group 1 (18-24 y.o.) ( $p=0.037$, AUC: 0.821 ; sensitivity: $88 \%$ and specificity: $71 \%$ for a cutoff of $209.50 \mathrm{ng} / \mathrm{ml})$. c ROC curve to diagnose MDD in group 2 (25-34 y.0.) ( $p<0.0001$, AUC: 0.855 ; sensitivity: $86 \%$ and specificity: $69 \%$ for a cutoff of $185.50 \mathrm{ng} / \mathrm{ml})$. d ROC curve to diagnose MDD in group 3 (35-44 y.0.) (p<0.0001, AUC: 0.975; sensitivity: $84 \%$ and specificity: $100 \%$ for a cutoff of $178 \mathrm{ng} / \mathrm{ml}$ ). e ROC curve to diagnose MDD in group 4 (45-54 y.o.) ( $p=0.007$, AUC: 0.821; sensitivity: $85 \%$ and specificity: $75 \%$ for a cutoff of 176.00$)$. f ROC curve to diagnose MDD in group 4 (55-65 y.0.) ( $p=0.16$, AUC: 0.750$)$. AUC: area under the ROC curve; ROC: receiver-operating characteristic.

MDD: $n=22$ ); Group 3-35-44 years old (HC: $\mathrm{n}=8$, MDD: $n=25$ ); Group 4-45-54 years old (HC: $n=12$, MDD: $n=13$ ); Group 5-55-65 years old (HC: $n=4$, MDD: $n=10)$. We established that IGF-1 had a good diagnostic value for predicting MDD in groups 1,2 , and 4; and excellent discriminatory power in Group 3. On the contrary, serum IGF-1 level exhibited poor value for the diagnosis of MDD in persons older than 55 years (Group 5). The cutoff value of IGF-1 for groups 1-4 decreased with age. Thus, in Group 1 it was $210 \mathrm{ng} / \mathrm{ml}$ (sensitivity $88 \%$; specificity $71 \%$ ); in Group $2-186 \mathrm{ng} / \mathrm{ml}$ (sensitivity 86\%; specificity 69\%); in Group 3-178 ng/ml (sensitivity $84 \%$; specificity $100 \%$ ); in Group $4-176 \mathrm{ng} / \mathrm{ml}$ (sensitivity $85 \%$; specificity $75 \%$ ).

Associations of IGF-1 concentrations with MDD symptoms Multiple linear regression models were performed to explore potential relationships between IGF-1 levels and different symptoms of a DE (Table 3). All four models showed that among MADRS items, only reported sadness, inner tension, and concentration difficulties were significantly positively associated with serum IGF-1 concentrations when adjusted for other covariates, even though there was a prominent negative association between age and IGF-1 levels. Moreover, the magnitude of the association with hypothymia was the highest. No other significant correlations between serum IGF-1 and MDD features were observed.

\section{Association of IGF-1 and cognitive functioning in the whole sample}

In addition, multiple linear regression models were performed to explore potential relationships between IGF-1 levels and the performance of cognitive tests by three separate linear regression analyses adjusted for relevant confounders (Table 4). Higher IGF-1 levels were significantly associated with the poor performance of all cognitive tests when adjusted for demographic covariates. Nevertheless, this association remained significant only between IGF-1 levels and DSST scores, when also adjusted for severity, number, and duration of the current DE (standardized $\beta=-0.21, p=0.03$ ). 
Table 3 Multiple linear regression models of specific relationships between IGF-1 $(\mathrm{ng} / \mathrm{ml})$ serum levels and MADRS symptoms $(n=$ $125)^{a}$

\begin{tabular}{|c|c|c|c|c|c|c|c|c|}
\hline \multirow[t]{2}{*}{ Variables } & \multicolumn{2}{|l|}{ Model 1} & \multicolumn{2}{|l|}{ Model 2} & \multicolumn{2}{|l|}{ Model 3} & \multicolumn{2}{|l|}{ Model 4} \\
\hline & $\overline{\beta(S E)}$ & St. $\beta$ & $\overline{\beta(S E)}$ & St. $\beta$ & $\overline{\beta(S E)}$ & St. $\beta$ & $\overline{\beta(S E)}$ & St. $\beta$ \\
\hline Age, years & $-1.81(0.69)$ & $-0.22^{*}$ & $-1.72(0.69)$ & $-0.21 *$ & $-1.80(0.69)$ & $-0.22 *$ & $-1.72(0.69)$ & $-0.21^{*}$ \\
\hline Gender & $27.10(17.52)$ & 0.13 & $25.81(17.94)$ & 0.13 & $26.37(17.48)$ & 0.13 & $24.29(17.89)$ & 0.12 \\
\hline Education, years & $4.13(3.98)$ & 0.08 & $4.61(3.97)$ & 0.09 & $3.82(3.98)$ & 0.08 & $4.23(3.96)$ & 0.09 \\
\hline MADRS total score & $-15.79(8.45)$ & -2.26 & $-13.78(8.54)$ & -1.97 & $-16.27(8.44)$ & -2.33 & $-14.05(8.50)$ & -2.01 \\
\hline \multicolumn{9}{|l|}{ MADRS items: } \\
\hline Apparent sadness & $-1.78(16.54)$ & -0.03 & $-2.85(16.45)$ & -0.05 & $0.73(16.61)$ & 0.01 & $-0.24(16.47)$ & -0.004 \\
\hline Reported sadness & 46.96 (17.79) & $0.92^{*}$ & $45.64(17.73)$ & $0.89^{*}$ & $45.90(17.76)$ & $0.90 *$ & $44.19(17.68)$ & $0.87^{*}$ \\
\hline Inner tension & 32.85 (10.09) & $0.55^{*}$ & $30.62(10.16)$ & $0.51 *$ & $33.49(10.07)$ & $0.56^{*}$ & $31.03(10.12)$ & $0.51 *$ \\
\hline Reduced sleep & $13.57(10.31)$ & 0.26 & $11.11(10.33)$ & 0.21 & $15.04(10.34)$ & 0.29 & $12.52(10.33)$ & 0.24 \\
\hline Reduced appetite & $14.88(12.08)$ & 0.24 & $12.86(12.05)$ & 0.21 & $15.45(12.06)$ & 0.25 & $13.34(11.99)$ & 0.21 \\
\hline Concentration difficulties & $29.52(11.68)$ & $0.51 *$ & $27.43(11.96)$ & $0.47^{*}$ & $28.79(11.67)$ & $0.50 *$ & $26.11(11.94)$ & $0.45^{*}$ \\
\hline Lassitude & $-5.56(13.34)$ & -0.10 & $-8.99(13.43)$ & -0.16 & $-4.97(13.31)$ & -0.09 & $-8.70(13.37)$ & -0.16 \\
\hline Inability to feel & $8.13(13.35)$ & 0.14 & $3.58(13.72)$ & 0.06 & $6.73(13.36)$ & 0.12 & $1.41(13.74)$ & 0.03 \\
\hline Pessimistic thoughts & 30.71 (13.29) & $0.51 *$ & $27.72(13.48)$ & $0.46^{*}$ & $29.62(13.28)$ & $0.49 *$ & $26.02(13.47)$ & 0.43 \\
\hline Suicidal thoughts & $10.36(12.21)$ & 0.11 & $11.01(12.13)$ & 0.11 & $13.18(12.38)$ & 0.14 & $14.26(12.29)$ & 0.15 \\
\hline Number of DE & & - & $7.39(6.17)$ & 0.12 & & - & $8.47(6.19)$ & 0.13 \\
\hline Duration of DE, weeks & & - & & - & $0.225(0.18)$ & 0.1 & $0.25(0.18)$ & 0.12 \\
\hline
\end{tabular}

${ }^{a}$ IGF-1 level was entered as a dependent outcome and all MADRS symptoms together in the same model as independent variables, along with increasing confounder adjustment. $\beta$ (SE) and standardized $\beta$ are presented. Model $1=$ adjusted for age, gender, education, and depression severity (according to MADRS); Model $2=$ Model $1+$ number of the DE; Model $3=$ Model $1+$ duration of the DE; Model $4=$ Model $1+$ number + duration of the DE; SE, standard error; ${ }^{*} p<0.05$

\section{The effect of antidepressant treatment on serum IGF-1 levels}

After 8 weeks of vortioxetine treatment in 48 MDD patients, we revealed that the intake of the antidepressant significantly decreased serum IGF-1 concentrations and improved clinical parameters of patients (Table 5). Thus, we observed a significant decrease in depression severity and improvement of cognitive impairment (measured as subjectively as objectively).

\section{Discussion}

In our study, we revealed that MDD patients had significantly higher serum levels of IGF-1 in comparison with HC. The most significant relationships were found between peripheral IGF-1 elevation and several DE symptoms - hypothymia, anxiety, and cognitive dysfunction. Eight weeks' vortioxetine treatment significantly attenuated serum IGF-1 concentrations and improved all psychopathological and neuropsychological parameters.

Regarding the peripheral IGF-1 increase in MDD patients, our findings are in line with some previous reports [21-26]. Nevertheless, the specific aspect of our study was that we obtained for the first time the cutoff values of serum IGF-1 to separate MDD patients from $\mathrm{HC}$ in different age groups, taking into account the proven fact of its decrease with age. Because of the small

Table 4 Multiple linear regression models of the associations between serum IGF-1 levels (ng/ml) and cognitive tests` performance $(n=125)^{\text {a }}$

\begin{tabular}{|c|c|c|c|c|c|c|}
\hline \multirow[t]{2}{*}{ Variables } & \multicolumn{2}{|l|}{ Model 1} & \multicolumn{2}{|l|}{ Model 2} & \multicolumn{2}{|l|}{ Model 3} \\
\hline & $\beta(S E)$ & St. $\beta$ & $\beta$ (SE) & St. $\beta$ & $\beta(S E)$ & St. $\beta$ \\
\hline RAVLT immediate recall & $-3.55(0.87)$ & $-0.35^{* *}$ & $-1.47(1.10)$ & -0.14 & $-1.48(1.08)$ & -0.15 \\
\hline TMT-B & $1.29(0.34)$ & $0.34^{* *}$ & $0.63(0.38)$ & 0.17 & $0.52(0.38)$ & 0.14 \\
\hline DSST & $-2.93(0.71)$ & $-0.38^{* *}$ & $-1.77(0.76)$ & $-0.23^{*}$ & $-1.62(0.75)$ & $-0.21^{*}$ \\
\hline
\end{tabular}

${ }^{a}$ IGF-1 level was entered as a dependent outcome and each cognitive test score as independent variables, along with increasing confounder adjustment. $\beta$ (SE) and standardized $\beta$ are presented. Model $1=$ adjusted for age, gender, and education, Model $2=$ Model $1+$ depression severity (according to MADRS), Model $3=$ Model $2+$ number + duration of DE; SE, standard error; ${ }^{*} p<0.05 ;{ }^{* *} p<0.0001$ 
Table 5 Changes in IGF-1 concentrations and different tests' scores after vortioxetine treatment

\begin{tabular}{|c|c|c|c|c|}
\hline & $\begin{array}{l}\text { At baseline } \\
n=48\end{array}$ & $\begin{array}{l}\text { After treatment } \\
n=48\end{array}$ & Mean change & $p$ \\
\hline \multicolumn{5}{|l|}{ Psychopathological symptoms } \\
\hline MADRS total score & $29(22-33)$ & $6(3-11)$ & 20.8 & $<0.0001$ \\
\hline CGI-S score & $4(4-5)$ & $1(1-2)$ & 2.3 & $<0.0001$ \\
\hline \multicolumn{5}{|c|}{ Patient-reported cognitive symptoms } \\
\hline PDQ-5 total score & $7(4-10)$ & $2(1-3)$ & 4.7 & $<0.0001$ \\
\hline \multicolumn{5}{|l|}{ Performance-based cognition } \\
\hline RAVLT immediate recall & $51(43-55)$ & $68(64-72)$ & -15.9 & $<0.0001$ \\
\hline TMT-B (s) & $75(63-96)$ & $47(36-60)$ & 30.1 & $<0.0001$ \\
\hline DSST & $54(42-61)$ & $62(51-71)$ & -9.7 & $<0.0001$ \\
\hline IGF-1 level (ng/ml) & $236(184-316)$ & $170(132-210)$ & 89.0 & $<0.0001$ \\
\hline
\end{tabular}

Data are presented as median (upper-lower quartile); $p$ according to Wilcoxon test (paired samples)

size of the age subgroups in our study, further work is needed to confirm these data.

The identification of peculiar DE symptoms having significant associations with IGF-1 levels was another distinctive feature of our study. Those were hypothymia, anxiety, and cognitive disturbances (particularly in executive functioning). This fact may indicate a pathogenic or compensatory role of IGF-1 for the development of the mentioned symptoms. The relationships between blood IGF-1 concentrations and cognitions in MDD have been previously studied only in the elderly population, and the results were contradictory to ours. Thus, Rueda et al. found a positive correlation with IGF-1 for cognition in women after adjustment for depression [27], a fact that was also reported in another study in healthy men [28]. Furthermore, decreased plasma IGF-1 levels were significantly associated with the decline of cognitive functioning and higher prevalence of depressive symptoms [29], whereas higher levels of IGF-1 were seen in those with improved cognition [30]. This inverse association found in elderly people may be explained by somatopause, which means that the secretion of IGF-1 decreases with advancing age in healthy adults [26, 31, 32].

The revealed discrepancies between IGF-1 increase in younger MDD patients and a decrease in older depressed individuals may indicate the unequal pathogenic role of the neurotrophin in these age-related cohorts. Reduction of cerebral and peripheral IGF-1 expression in the elderly may be directly related to the impairments in emotional and cognitive processing resulting in mood and neuropsychological symptoms. At the same time, the enhancement of the peripheral IGF-1 expression in young patients with MDD may be a compensatory mechanism in response to its brain synthesis decrease [25]. Also, the elevation of peripheral IGF-1 concentrations may be explained by the decreased cerebral bioavailability of the neurotrophin due to the reduced sensitivity of IGF-1 receptors under the neuroinflammatory stress [26]. Previously we have suggested that the activity of the cerebral-hepatic axis (GH/IGF-1 axis) increases in response to insufficient concentrations of this neurotrophin in the brain [33]. As a result, the production of IGF-1 in the liver elevates. When the production of IGF-1 in the brain is restored (i.e., after antidepressant treatment), the hepatic IGF-1 production and its blood concentration decline.

This suggestion is supported by the fact that IGF-1 concentrations in cerebrospinal fluid were found to be lower in MDD persons compared to $\mathrm{HC}$ and normalized after various antidepressant treatments irrespective of the class of medication, duration of therapy or response [34].

Data on a significant decrease of serum IGF-1 concentrations under vortioxetine treatment obtained in the study are consistent with the proposed concept and supported by several previous investigations. It was demonstrated that antidepressants - amitriptyline, doxepin, fluoxetine, paroxetine - led to a significant decline of peripheral IGF-1 levels [21, 35, 36]. For vortioxetine, such evidence was obtained for the first time to our knowledge. According to our hypothesis [33], we can assume that vortioxetine treatment restored cerebral IGF-1 concentrations and, consequently, decreased its compensatory production by the liver. Summarizing the evidence that a compensatory increase of peripheral IGF-1 in response to a deficiency in the brain may have pathogenetic effects for accelerating aging [33], we demonstrated that vortioxetine might have protective mechanisms against progeric effects, in particular telomerase attrition. As it was shown that IGF-1 induces the intracellular kinase-dependent pathways involved in proliferative processes and telomere attrition in various organs, which can be considered as the underlying mechanism of aging [37]. Nevertheless, the direct effect of vortioxetine on longevity needs further investigation. 


\section{Conclusion}

In conclusion, taken together, in the present study of the Ukrainian cohort, higher serum IGF-1 concentrations were associated with MDD status (in the age cohort of 18-54 years old), the severity of hypothymia, anxiety, and cognitive decline (predominantly executive dysfunction). This may indicate a pathogenic role of IGF-1 for the mentioned symptoms development. Treatment with vortioxetine substantially attenuated serum IGF-1 levels and improved all psychopathological and neuropsychological parameters. Further studies are needed to examine the relationships between IGF-1 and other neurotrophic factors in MDD patients and their influence on separate MDD symptoms.

\section{Abbreviations}

AUC-ROC: Areas under the receiver operating characteristic curves; CGII: Clinical Global Impression Improvement; CGI-S: Clinical Global Impression Severity; DE: Depressive episode; DSM-5: Diagnostic and Statistical Manual of Mental Disorders, Fifth Edition; DSST: Digit Symbol Substitution Test; IGF1: Insulin-like growth factor-1; HC: Healthy controls; MADRS: MontgomeryAsberg Depression Rating Scale; MDD: Major depressive disorder; NNM: Number Needed to Misdiagnose; PDQ-5: Perceived Deficit Questionnaire-5; RAVLT: Rey Auditory Verbal Learning Test; ROC: Receiveroperating characteristic; SD: Standard deviation; SPSS: Statistical Package for the Social Sciences; TMT-B: Trail Making Test B

\section{Acknowledgments}

We are grateful to Viktoria $V$. Levada for substantial help during data collection and all participants of the study.

\section{Authors`contributions}

OL supervised the study. OL and AT were involved in the concept of the research and collection of the data. OL, AT, IP contributed to data preparation and literature search. OL and AT analyzed the data and wrote the first draft of the manuscript. OL, AT, IP authors critically revised the draft and approved the final version of the manuscript.

\section{Funding}

No specific funding was provided for this study.

\section{Availability of data and materials}

The datasets used and/or analyzed during the current study are available from the corresponding author on reasonable request.

\section{Ethics approval and consent to participate}

Ethics approval was obtained from the Zaporizhzhia Medical Academy of Postgraduate Education Ethics Committee - Zaporizhzhia, Ukraine, on 15th September 2016. All participants provided written informed consent.

\section{Consent for publication}

Not applicable.

\section{Competing interests}

O.A. Levada is a member of advisory and/or speaker boards of the following companies: Lundbeck, Pfizer, Acino. A.S. Troyan gave presentations for Lundbeck and Acino.

\section{Author details}

'State Institution "Zaporizhzhia Medical Academy of Postgraduate Education Ministry of Health of Ukraine", 20 Winter Boulevard, Zaporizhzhia 69096, Ukraine. ${ }^{2}$ Institute of Psychiatry of Taras Shevchenko National University of Kyiv, 64 Volodymirskaya Street, Kyiv 01033, Ukraine.
Received: 2 May 2019 Accepted: 30 April 2020

Published online: 08 May 2020

\section{References}

1. Smith K. Mental health: a world of depression. Nature. 2014;515(7526):181.

2. Kessler RC, Berglund P, Demler O, Jin R, Koretz D, Merikangas KR, et al. The epidemiology of major depressive disorder: results from the National Comorbidity Survey Replication (NCS-R). JAMA. 2003;289(23):3095-105.

3. Vandeleur CL, Fassassi S, Castelao E, Glaus J, Strippoli MF, Lasserre AM, et al. Prevalence and correlates of DSM-5 major depressive and related disorders in the community. Psychiatry Res. 2017;250:50-8.

4. American Psychiatric Association. Diagnostic and statistical manual of mental disorders. 5th ed. Arlington: American Psychiatric Association; 2013.

5. Duman RS. Neurobiology of stress, depression, and rapid acting antidepressants: remodeling synaptic connections. Depress Anxiety. 2014;31:4.

6. Li Y, Pehrson AL, Waller JA, Dale E, Sanchez C, Gulinello M. A critical evaluation of the activity-regulated cytoskeleton-associated protein (Arc) Arg3.1)'s putative role in regulating dendritic plasticity, cognitive processes, and mood in animal models of depression. Front Neurosci. 2015;9:279.

7. Darcet F, Gardier AM, Gaillard R, David DJ, Guilloux JP. Cognitive dysfunction in major depressive disorder. A translational review in animal models of the disease. Pharmaceuticals (Basel). 2016;9(1):E9.

8. Rial D, Lemos C, Pinheiro H, Duarte JM, Gonçalves FQ, Real Jl, et al. Depression as a glial-based synaptic dysfunction. Front Cell Neurosci. 2016;9:521.

9. Sweatt JD. Neural plasticity and behavior — sixty years of conceptual advances. J Neurochem. 2016:139(Suppl 2):179-99.

10. Duman RS. Role of neurotrophic factors in the etiology and treatment of mood disorders. NeuroMolecular Med. 2004;5(1):11-25.

11. Annunziata M, Granata R, Ghigo E. The IGF system. Acta Diabetol. 2011;48(1):1-9.

12. Russo VC, Gluckman PD, Feldman EL, Werther GA. The insulin-like growth factor system and its pleiotropic functions in brain. Endocr Rev. 2005;26(7): 916-43.

13. Szczesny E, Slusarczyk J, Glombik K, Budziszewska B, Kubera M, Lason W, et al. Possible contribution of IGF-1 to depressive disorder. Pharmacol Rep. 2013;65(6):1622-31.

14. Levada OA, Troyan AS. Insulin-like growth factor-1: a possible marker for emotional and cognitive disturbances, and treatment effectiveness in major depressive disorder. Ann General Psychiatry. 2017;16:38.

15. Levada OA, Troyan AS. Cognitive-functional relationships in major depressive disorder: crucial data from a Ukrainian open-label study of vortioxetine versus escitalopram. J Affect Disord. 2019;250:114-22.

16. Montgomery SA, Åsberg M. A new depression scale designed to be sensitive to change. Br J Psychiatry. 1979;134:382-9.

17. Guy W. Clinical Global Impressions. In: Guy W, editor. ECDEU assessment manual for psychopharmacology revised. Rockville: National Institute of Mental Health; 1976. p. 217-22

18. Cha D. Perceived deficits questionnaire - depression, 5-item (PDQ-D-5). In McIntyre R, editor. Cognitive impairment in major depressive disorder: clinical relevance, biological substrates, and treatment opportunities. Cambridge: Cambridge University Press; 2016. p. 242-56.

19. Strauss E, Sherman EMS, Spreen O. A compendium of neuropsychological tests: administration, norms, and commentary. Oxford, England: Oxford University Press; 2006.

20. Habibzadeh F, Yadollahie M. Number needed to misdiagnose: a measure of diagnostic test effectiveness. Epidemiology. 2013;24(1):170.

21. Deuschle M, Blum WF, Strasburger CJ, Schweiger U, Weber B, Körner A, et al. Insulin-like growth factor-I (IGF-I) plasma concentrations are increased in depressed patients. Psychoneuroendocrinology. 1997;22:493-503.

22. Lesch KP, Rupprecht $R$, Müller U, Pfüller H, Beckmann H. Insulin-like growth factor I in depressed patients and controls. Acta Psychiatr Scand. 1988;78: $684-8$.

23. Franz B, Buysse DJ, Cherry CR, Gray NS, Grochocinski VJ, Frank E, et al. Insulin-like growth factor 1 and growth hormone binding protein in depression: a preliminary communication. J Psychiatr Res. 1999;33:121-7.

24. Kopczak A, Stalla GK, Uhr M, Lucae S, Hennings J, Ising M, et al. IGF-I in major depression and antidepressant treatment response. Eur Neuropsychopharmacol. 2015;25:864-72.

25. Bot M, Milaneschi Y, Penninx BW, Drent ML. Plasma insulin-like growth factor I levels are higher in depressive and anxiety disorders, but lower in antidepressant medication users. Psychoneuroendocrinology. 2016;68: 148-55. 
26. Tu KY, Wu MK, Chen YW, Lin PY, Wang HY, Wu CK, et al. Significantly Higher Peripheral Insulin-Like Growth Factor-1 Levels in Patients With Major Depressive Disorder or Bipolar Disorder Than in Healthy Controls: A MetaAnalysis and Review Under Guideline of PRISMA. Medicine (Baltimore). 2016; 95:e2411.

27. Rueda Alfaro S, Serra-Prat M, Palomera E, Falcon I, Cadenas I, Boquet X, et al. Hormonal determinants of depression and cognitive function in independently-living elders. Endocrinol Nutr. 2008;55:396-401.

28. Aleman A, Verhaar HJ, De Haan EH, De Vries WR, Samson MM, Drent ML, et al. Insulin-like growth factor-l and cognitive function in healthy older men. J Clin Endocrinol Metab. 1999:84:471-5.

29. Lin F, Suhr J, Diebold S, Heffner KL. Associations between depressive symptoms and memory deficits vary as a function of insulin-like growth factor (IGF-1) levels in healthy older adults. Psychoneuroendocrinology. 2014;42:118-23.

30. Palomino A, Gonzalez-Pinto A, Martinez-Cengotitabengoa M. Ruiz de Azua $\mathrm{S}$, Alberich S, Mosquera F, et al. relationship between negative symptoms and plasma levels of insulin-like growth factor 1 in first-episode schizophrenia and bipolar disorder patients. Prog Neuro-Psychopharmacol Biol Psychiatry. 2013;44:29-33.

31. Corpas E, Harman SM, Blackman MR. Human growth hormone and human aging. Endocr Rev. 1993;14:20-39.

32. Bartke A. Growth hormone and aging: a challenging controversy. Clin Interv Aging. 2008;3:659-65.

33. Levada OA, Troyan AS. Major depressive disorder and accelerated aging from a peripheral IGF-1 overexpression perspective. Med Hypotheses. 2020; 138:109610.

34. Schilling C, Blum WF, Heuser I, Paslakis G, Wudy SA, Deuschle M. Treatment with antidepressants increases insulin-like growth factor-I in cerebrospinal fluid. J Clin Psychopharmacol. 2011;31(3):390-2.

35. Weber-Hamann B, Blum WF, Kratzsch J, Gilles M, Heuser I, Deuschle M. Insulin-like growth factor-I (IGF-I) serum concentrations in depressed patients: relationship to saliva cortisol and changes during antidepressant treatment. Pharmacopsychiatry. 2009;42:23-8.

36. Sharma AN. da Costa e Silva BF, Soares JC, Carvalho AF, Quevedo J. role of trophic factors GDNF, IGF-1 and VEGF in major depressive disorder: a comprehensive review of human studies. J Affect Disord. 2016;197:9-20.

37. Taya S, Inagaki N, Sengiku H, Makino H, Iwamatsu A, Urakawa I, et al. Direct interaction of insulin-like growth factor-1 receptor with leukemia-associated RhoGEF. J Cell Biol. 2001;155(5):809-20.

\section{Publisher's Note}

Springer Nature remains neutral with regard to jurisdictional claims in published maps and institutional affiliations.

Ready to submit your research? Choose BMC and benefit from:

- fast, convenient online submission

- thorough peer review by experienced researchers in your field

- rapid publication on acceptance

- support for research data, including large and complex data types

- gold Open Access which fosters wider collaboration and increased citations

- maximum visibility for your research: over $100 \mathrm{M}$ website views per year

At $\mathrm{BMC}$, research is always in progress.

Learn more biomedcentral.com/submissions 